Article

\title{
Effective 5G Wireless Downlink Scheduling and Resource Allocation in Cyber-Physical Systems ${ }^{\dagger}$
}

\author{
Ankur Vora $₫$ and Kyoung-Don Kang * \\ Computer Science Department, State University of New York at Binghamton, Binghamton, NY 13902, USA; \\ avora4@binghamton.edu \\ * Correspondence: kang@binghamton.edu \\ † This paper is an extended version of our paper published in IEEE 5G World Forum (5GWF), Santa Clara, CA, \\ USA, 9-11 July 2018.
}

Received: 15 October 2018; Accepted: 12 November 2018; Published: 15 November 2018

\begin{abstract}
In emerging Cyber-Physical Systems (CPS), the demand for higher communication performance and enhanced wireless connectivity is increasing fast. To address the issue, in our recent work, we proposed a dynamic programming algorithm with polynomial time complexity for effective cross-layer downlink Scheduling and Resource Allocation (SRA) considering the channel and queue state, while supporting fairness. In this paper, we extend the SRA algorithm to consider 5G use-cases, namely enhanced Machine Type Communication (eMTC), Ultra-Reliable Low Latency Communication (URLLC) and enhanced Mobile BroadBand (eMBB). In a simulation study, we evaluate the performance of our SRA algorithm in comparison to an advanced greedy cross-layer algorithm for eMTC, URLLC and LTE (long-term evolution). For eMTC and URLLC, our SRA method outperforms the greedy approach by up to $17.24 \%, 18.1 \%, 2.5 \%$ and $1.5 \%$ in terms of average goodput, correlation impact, goodput fairness and delay fairness, respectively. In the case of LTE, our approach outperforms the greedy method by $60 \%, 2.6 \%$ and $1.6 \%$ in terms of goodput, goodput fairness and delay fairness compared with tested baseline.
\end{abstract}

Keywords: 5G wireless technology; massive multiple-input-multiple-output (MIMO) communications; scheduling and resource allocation (SRA); orthogonal frequency division multiplexing (OFDM); filter bank multi-carrier (FBMC)

\section{Introduction}

Cyber-Physical Systems (CPS) and the Internet of Things (IoT) support numerous important applications, such as connected cars, factory automation, intelligent surveillance, smart homes and smart agriculture. The total number of IoT devices has already exceeded seven billion in the second quarter of 2018 without including two billion smartphones in the world [1]. It is projected that there will be approximately 50 billion connected devices by the end of 2020 [2]. Further, 70\% of IoT devices will use cellular technology with better connectivity and reliability [3]. Thus, massive cellular traffic and connectivity requirements should be handled gracefully, dramatically increasing the demand for higher wireless communication performance and enhanced connectivity. It is difficult to support the demand using today's wireless technology [3,4].

To address these challenges, the International Telecommunication Union (ITU) and International Mobile Telecommunication (IMT) have envisioned the fifth generation of cellular communication technology, called 5G for brevity. A 5G base station needs to support efficient Scheduling and Resource Allocation (SRA) via Time Division Duplexing (TDD), as well as Frequency Division Duplexing (FDD) [5-7]. In 5G, user applications are classified into three broad categories: (1) enhanced Mobile Broadband (eMBB), (2) enhanced Machine Type Communication (eMTC) and (3) Ultra-Reliable Low 
Latency Communication (URLLC) [8]. To support different 5G use-cases, as illustrated in Figure 1, a base station needs to use different numbers of Resource Blocks (RBs) along the time axis for TDD and different sub-carrier bandwidth allocations on the frequency axis to support FDD. For eMBB, typically, a $100-\mathrm{MHz}$ bandwidth in the frequency domain and at least $500 \mathrm{RBs}$ in the time domain are needed. For URLLC, the latency and reliability are critical. It uses a modest bandwidth up to $5 \mathrm{MHz}$ with an RB sequence of 25 symbols for FDD and TDD, respectively. In addition, eMTC uses $1.4 \mathrm{MHz}$ bandwidth and six RBs in the frequency and time domain, respectively.

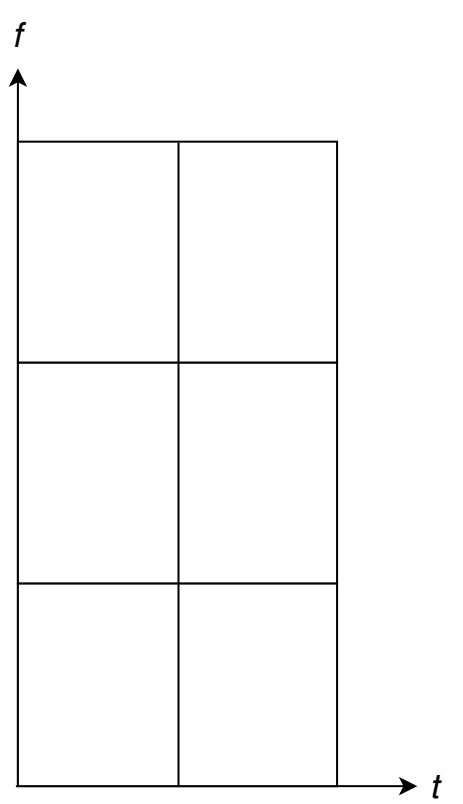

eMBB

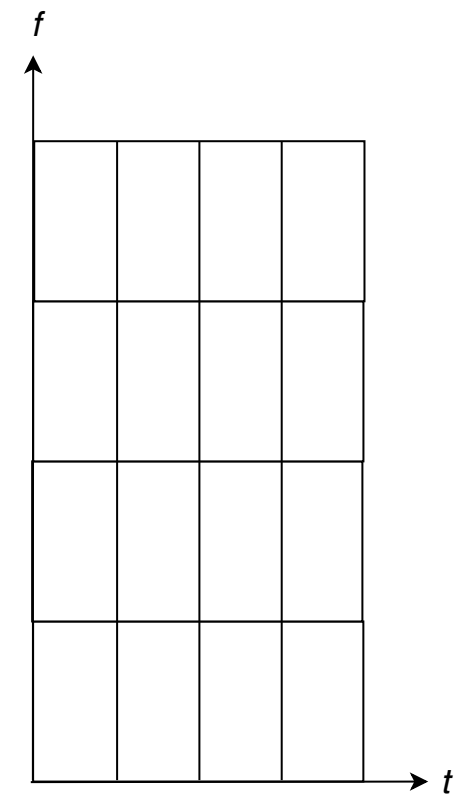

URLLC

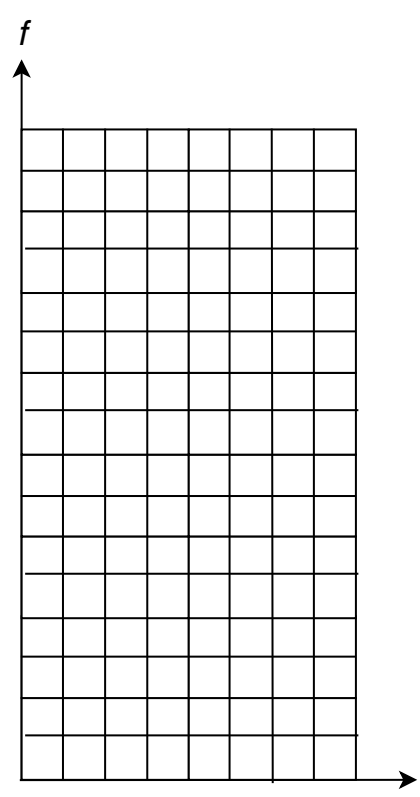

eMTC

Figure 1. International Mobile Telecommunication (IMT)-2020 use-cases from Frequency Division Duplexing (FDD)/Time Division Duplexing (TDD) perspectives. eMBB, enhanced Mobile Broadband; URLLC, Ultra-Reliable Low Latency Communication; eMTC, enhanced Machine Type Communication.

These use-cases show that a 5G base station needs to handle diverse traffic at each transmission interval. It is challenging to schedule and allocate resources at each transmission interval for many devices with diverse use-cases. In addition, the first generation of 5G comes as a Non-Stand-Alone (NSA) architecture that requires backward compatibility with the previous generation Long-Term Evolution (LTE) technology [9-12], making Scheduling and Resource Allocation (SRA) even more challenging.

To address the challenges of 5G, Feminias et al. [13] have recently proposed a novel cross-layer SRA framework by extending their previous work [14]. In their work, the utility function is defined in terms of the weighted goodput for cross-layer SRA. It provides a greedy cross-layer optimization over the Physical (PHY) and Data Link Control (DLC) layers to support effective SRA for massive Multiple-Input-Multiple-Output (MIMO) systems by taking advantage of higher bandwidth [15] and adaptive Modulation and Coding Schemes (MCSs) [16]. However, their SRA algorithm is greedy, potentially producing sub-optimal results; hence, it may not perform well for broader $5 \mathrm{G}$ use-cases. To address this issue, we have designed a new SRA algorithm based on dynamic programming [17]. It formulates the utility function based on the available bandwidth and required RBs, while allocating resources to maximize the total utility. Although the knapsack-like problem of dynamic SRA is usually NP-hard, we show that the time complexity of our algorithm is polynomial in a practical sense. In this paper, we further extend our conference publication [17] as follows: 
- This paper discusses the need for SRA based on dynamic programming at the base station to support diverse 5G use-cases, as depicted in Figure 1.

- We extend our SRA algorithm for 5G use-cases: eMTC, URLLC and eMBB. The problem formulation shows the scalability of the utility function to adopt all these use-cases with LTE to support the first generation 5G NSA architecture.

- An extended discussion of related work is given in Section 2 to review state-of-the-art SRA techniques and discuss the need for our work presented in this paper.

- In this paper, we extend the performance evaluation to consider the eMTC and URLLC in addition to LTE. For LTE, our SRA algorithm outperforms the greedy approach [13] by up to $60 \%, 2.6 \%$ and $1.6 \%$ in terms of goodput, goodput fairness and delay fairness, conforming to [17]. For eMTC and URLLC associated with more demanding performance requirements, our SRA algorithm continues to outperform the greedy cross-layer approach [13] by up to $17.24 \%, 18.1 \%$, $2.5 \%$ and $1.5 \%$ in terms of average goodput, correlation impact, goodput fairness and delay fairness, respectively.

The remainder of this paper is organized as follows. Section 2 reviews state-of-the-art SRA algorithms. Section 3 formulates the SRA problem. In Section 4, our SRA algorithm is described and its time complexity analyzed. In Section 5, the performance of the proposed SRA algorithm is evaluated in comparison to [13] for LTE, eMTC and URLLC use-cases. Finally, the paper is concluded and future work is discussed in Section 6.

\section{Related Work}

In a mobile network, when a user requests data from the Internet, the request is sent to the base station. The base station retrieves the data through the Internet and provides them to the user in the form of data packets. These data packets are framed into larger data frames and transmitted from the base station towards the User Equipment (UE). These data frames consist of time and frequency resources in terms of RBs and the number of subcarriers, respectively. Allocating resources to multiple users in a single data frame is known as the SRA problem at the base station. SRA has been studied over the decades from a variety of performance perspectives: (1) spectral efficiency, (2) scalability, (3) computational complexity, (4) Quality of Service (QoS), (5) fairness, (6) target delay, (7) queue length, (8) priority, (9) Guaranteed Bit-Rate (GBR), etc. [18-21]. In this paper, we classify state-of-the-art approaches for SRA into two broad categories: (1) channel-dependent and (2) channel-independent SRA algorithms, as summarized in Table 1. Within each category, they are further classified into subcategories.

All the algorithms in Table 1 are used to handle either a single type of traffic or multiple QoS classes at the base station. In the case of 5G, SRA decisions take place based on the queue and Channel State Information (CSI); hence, the channel-independent SRA algorithms are not relevant. In the case of channel-dependent algorithms, most work has been focused on supporting a GBR and dealing with delay-sensitive traffic. However, in the case of 5G, the base station needs a cross-layer SRA algorithm, which can make cross-layer SRA decisions considering the queue and CSI. Table 1 shows the state-of-the-art system-centric cross-layer SRA algorithms that make SRA decisions considering both the queue and channel state. However, none of them has specifically been designed from the $5 \mathrm{G}$ perspective. In $5 \mathrm{G}$, the base station needs to serve diverse traffic of different use-cases in each transmission interval. An advanced cross-layer approach [13] considers the queue state and CSI together for SRA decisions; however, it is a greedy algorithm that may produce suboptimal results. To address this problem, we have proposed a new cross-layer SRA algorithm based on dynamic programming in [17] that makes optimal SRA decisions at 5G base stations with polynomial time complexity. In this paper, we extend our previous SRA framework [17] to accommodate various $5 \mathrm{G}$ use-cases. 
Table 1. Taxonomy of Scheduling and Resource Allocation (SRA) algorithms.

\begin{tabular}{|c|c|c|c|}
\hline Category & $\begin{array}{l}\text { Dependent } \\
\text { Parameter }\end{array}$ & Algorithm Name & Resource Allocation Summary \\
\hline \multirow{8}{*}{$\begin{array}{l}\text { Channel- } \\
\text { Independent }\end{array}$} & \multirow{6}{*}{$\begin{array}{l}\text { Classical } \\
\text { Algorithms }\end{array}$} & $\begin{array}{l}\text { Proportional } \\
\text { Fair (PF) [22-24] }\end{array}$ & $\begin{array}{l}\text { Allocate resources to users in proportion } \\
\text { to their weights }\end{array}$ \\
\hline & & $\begin{array}{l}\text { First-In-First- } \\
\text { Out (FIFO) [25-27] }\end{array}$ & $\begin{array}{l}\text { Allocate resources based on their arrival } \\
\text { order }\end{array}$ \\
\hline & & Round Robin [28-30] & $\begin{array}{l}\text { Allocate resource to each user for a fixed } \\
\text { time interval }\end{array}$ \\
\hline & & $\begin{array}{l}\text { Weighted Fair } \\
\text { Queuing [31,32] }\end{array}$ & $\begin{array}{l}\text { Allocate resources based on users' } \\
\text { weights inversely proportional to costs }\end{array}$ \\
\hline & & $\begin{array}{l}\text { Blind Equal } \\
\text { Throughput [33-35] }\end{array}$ & $\begin{array}{l}\text { Allocate resources to maintain } \\
\text { minimum throughput requirements }\end{array}$ \\
\hline & & $\begin{array}{l}\text { Largest Weighted } \\
\text { Delay First [36-38] }\end{array}$ & $\begin{array}{l}\text { Allocate resources based on users' } \\
\text { weights and delay sensitivities }\end{array}$ \\
\hline & VoIP & Delay sensitive [39-43] & $\begin{array}{l}\text { Prioritize VoIP traffic and provide } \\
\text { best effort service to other traffic }\end{array}$ \\
\hline & Video Streaming & $\begin{array}{l}\text { Dynamic Adaptive Streaming } \\
\text { Over HTTP (DASH) [44-47] }\end{array}$ & $\begin{array}{l}\text { Ensure a guaranteed bit-rate to high-rank } \\
\text { users based on the channel quality }\end{array}$ \\
\hline \multirow{12}{*}{$\begin{array}{l}\text { Channel- } \\
\text { Dependent }\end{array}$} & \multirow{3}{*}{$\begin{array}{l}\text { Guaranteed } \\
\text { Bit-Rate } \\
\text { (GBR) }\end{array}$} & Priority Based [48-51] & Allocate resources based on user priority \\
\hline & & $\begin{array}{l}\text { Quality of Service (QoS) Aware } \\
\text { Scheduler [52-54] }\end{array}$ & $\begin{array}{l}\text { Prioritize users and allocate resources } \\
\text { accordingly }\end{array}$ \\
\hline & & $\begin{array}{l}\text { Hybrid Schedulers } \\
{[55,56]}\end{array}$ & $\begin{array}{l}\text { Allocate resources based on users' QoS } \\
\text { and delay sensitivity requirements }\end{array}$ \\
\hline & \multirow{7}{*}{ Delay-Sensitive } & $\begin{array}{l}\text { Weighted Delay } \\
\text { First [57-59] }\end{array}$ & $\begin{array}{l}\text { Assign a higher weight and more } \\
\text { resources to a user close to its target }\end{array}$ \\
\hline & & $\begin{array}{l}\text { Hybrid Automatic Repeat Request } \\
\text { (HARQ) Aware Scheduling [60-62] }\end{array}$ & $\begin{array}{l}\text { Prioritize users based on the average } \\
\text { throughput and delay }\end{array}$ \\
\hline & & $\begin{array}{l}\text { Exponential/Proportional } \\
\text { Fair (Exp/PF) }[63,64]\end{array}$ & $\begin{array}{l}\text { Maximize throughput while providing } \\
\text { a fair level of services }\end{array}$ \\
\hline & & $\begin{array}{l}\text { Two-Level } \\
\text { Scheduler }[65,66]\end{array}$ & $\begin{array}{l}\text { Prioritize real-time and non-real-time } \\
\text { data to allocate resources }\end{array}$ \\
\hline & & $\begin{array}{l}\text { Delay-Prioritized } \\
\text { Scheduling }[38,67]\end{array}$ & $\begin{array}{l}\text { Assign resources based on users' delay } \\
\text { requirements }\end{array}$ \\
\hline & & Exp and Log Rule $[68,69]$ & $\begin{array}{l}\text { Assign resources to a user based on his/her } \\
\text { position in the queue }\end{array}$ \\
\hline & & $\begin{array}{l}\text { Game Theory-Based } \\
\text { Scheduling [70,71] }\end{array}$ & $\begin{array}{l}\text { Fairly distribute the resources among the } \\
\text { participating users based on game theory }\end{array}$ \\
\hline & \multirow{2}{*}{$\begin{array}{l}\text { Cross-Layer } \\
\text { Algorithm }\end{array}$} & $\begin{array}{l}\text { Overload-State Downlink } \\
\text { Resource Allocation [72-74] }\end{array}$ & $\begin{array}{l}\text { Assign resources based on the queue state } \\
\text { information }\end{array}$ \\
\hline & & $\begin{array}{l}\text { Greedy Resource Block (RB) } \\
\text { Allocation [13] }\end{array}$ & $\begin{array}{l}\text { Assign resources based on the queue and } \\
\text { channel state information }\end{array}$ \\
\hline
\end{tabular}

\section{Problem Formulation}

Figure 2 shows the downlink time-slotted architecture inspired by [13]. It consists of mainly two parts: a base station and User Equipment (UE), as shown in the figure. The base station has $N_{T}$ transmit antennas with transmitting power $P_{T}$. In a real environment, multiple mobile stations are connected with a base station. For simplicity, we show just one UE in the figure and assume that all the Mobile Stations $M S=\left\{M S_{1}, \ldots, M S_{N}\right\}$ follow the same architecture. Each of these mobile stations supports multiple-input-multiple-output (MIMO) technology with an array of $N_{T} \times N_{R}$ transmitting and receiving antennas. 
In this architecture, we have considered a busy base station with an infinite traffic queue: there is a continuous traffic flow from the upper layer to the DLC and PHY layers of the base station, as shown in Figure 2. Requests from different mobile stations are queued at the base station. Requests can consists of a variety of $5 \mathrm{G}$ use cases, such as eMBB, URLLC and eMTC. SRA decisions in this architecture are cross-layer in that decisions are made considering the DLC and PHY layers, as shown in Figure 2. It takes input from the queue state and the CSI, which is three-dimensional information consisting of time (symbols), frequency (number of sub-bands) and space (number of antennas), as shown in Figure 2. At each transmission interval, a CSI exchange takes place between the base station and a mobile station. The mobile station CSI tells the base station about the channel properties of a communication link such as delay-Doppler spread, Signal-Interference-to-Noise-Ratio (SINR), angle-of-arrival, angle-of-departure and PHY layer configurations such as Modulation and Coding Schemes (MCS) and the rank indicator, precoding matrix indicator and channel quality indicator received from the mobile station. The scheduled resources are then transferred to the PHY layer for transmission, as shown in the figure. At the PHY layer, the information goes through the Adaptive Modulation and Coding (AMC) process, which decides the appropriate modulation schemes for individual users. Data modulation is followed by MIMO processing and data transmission using transmit antennas $[14,75,76]$ in the base station. In a mobile station, the reverse process takes place: the PHY layer performs multi-carrier post-processing and MIMO equalization on the received signals through the receiving antennas. The AMC and MIMO precoding matrices are exchanged and known by the mobile station during the CSI exchange. Finally, the demodulation process extracts the coded data.

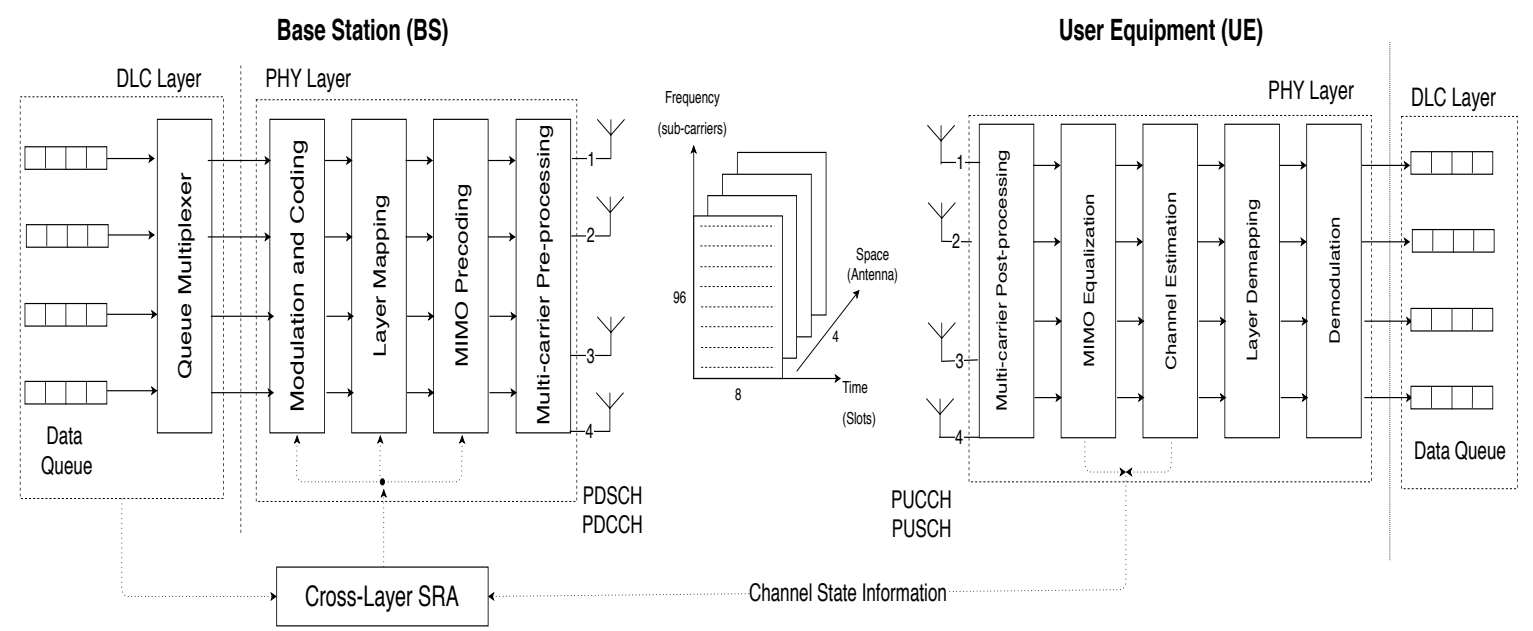

Figure 2. System architecture. DLC, Data Link Control; PDSCH (Physical Downlink Shared Channel); PDCCH (Physical Downlink Control Channel); PUCCH (Physical Uplink Control Channel); PUSCH (Physical Uplink Shared Channel).

In the case of multi-carrier time-slotted downlink architecture, resources, called RBs, are allocated in the time domain across multiple sub-bands. For each transmission time interval $t$, RBs consist of $N_{s y m}$ symbols for a duration $T_{p}$, along the time axis and sub-bands of $\Delta f=\frac{1}{T_{p}}$ in the frequency domain. On the time axis, each RB holds a fixed number of time slots $T_{s}^{P H Y}$. In this paper, PHY represents either orthogonal frequency division multiplexing (OFDM) or filter bank multi-carrier (FBMC) symbols.

\subsection{Orthogonal Frequency Division Multiplexing}

OFDM has been used over a decade and has proved its robustness in multi-carrier technologies, such as Wi-Fi and cellular technology. It uses multiple smaller subcarriers to avoid the Inter-Channel Interference (ICI) and Inter-Symbol Interference (ISI) over the network. It adds a Cyclic Prefix (CP) to demodulate the signal effectively on the receiver side. It is assumed that the transmitter and receiver are synchronized properly to avoid the misinterpretation of symbols [77]. It uses the Inverse Fast 
Fourier Transform (IFFT) to convert the symbol from the time domain to the frequency domain at the transmitter, while applying the Fast Fourier Transform (FFT) to transform the symbols from the frequency domain to the time domain at the receiver. Since OFDM uses the Cyclic Prefix (CP) to cancel out ISI, there are $N_{s y m}^{\text {long }}$ OFDM symbols prefixed with a long CP of duration $T_{C P}^{\text {long }}$. Furthermore, there are $N_{\text {sym }}^{\text {short }}=N_{\text {sym }}-N_{\text {sym }}^{\text {long }}$ symbols prefixed with a short CP of duration $T_{C P}^{\text {short }}$. Thus, for OFDM systems, the fixed time slot size is:

$$
T_{s}^{\text {OFDM }}=\left(N_{\text {sym }} \times T_{p}\right)+\left(N_{\text {sym }}^{\text {long }} \times T_{C P}^{\text {long }}\right)+\left(N_{\text {sym }}^{\text {short }} \times T_{C P}^{\text {short }}\right)
$$

where $T_{p}$ is the symbol duration and $T_{C P}$ is the symbol duration with CP. Hence, OFDM is spectrally inefficient since it adds $\left(N_{\text {sym }}^{\text {long }} \times T_{C P}^{\text {long }}\right)+\left(N_{\text {sym }}^{\text {short }} \times T_{C P}^{\text {short }}\right)$ cyclic prefixes, which consume additional resources in the frequency domain. The CPs usually consume about $25 \%$ of the subcarrier bandwidth $\Delta f[78]$.

\section{Filter Bank Multi-Carrier}

In 5G, a base station needs to serve a large number of users; thus, it needs a spectrally-efficient PHY waveform. FBMC uses a chain of filters at each subcarrier to make it spectrally efficient, unlike OFDM, which uses CPs. Hence, FBMC can enhance the spectral efficiency and improve the network performance. For FBMC, the fixed time slot size $T_{s}^{F B M C}$ is:

$$
T_{s}^{F B M C}=N_{s y m} \times T_{p}
$$

We assume that the SRA process happens at the beginning of a Transmission Time Interval (TTI) between two consecutive time slots, similar to [13]. By comparing Equations (1) and (2), we observe that FBMC achieves higher spectral efficiency compared to OFDM due to the absence of CPs. However, FBMC applies a filter chain at each individual subcarrier. Hence, the base station needs to spend more time and computational resources compared to OFDM.

\subsection{DLC Layer}

The queue $Q_{u}$ at the base station may contain a variety of traffic such as eMBB, URLLC and eMTC. At each TTI, the base station allocates a spatial stream, $L_{u}$, for each user $u$. The total transmission capacity at each TTI is $\gamma_{u, l}\left(t, N_{B_{u}}\right)$, where $l \epsilon L_{u}=\left\{1, \ldots, L_{u}\right\}$ and $N_{B_{u}}$ is the number of RBs required by user $u$. The total queue length at each TTI at the base station is:

$$
Q_{u}(t+1)=Q_{u}(t)+A_{u}(t)-S_{u}(t)
$$

where $A_{u}(t)$ and $S_{u}(t)$ represent the number of the arriving data bits to transmit for the user $u$ during TTI $t$ and that successfully transmitted to the user, respectively. These queues are then forwarded to the PHY layer for SRA.

\subsection{PHY Layer}

When there are $N_{M S}$ mobile stations, at the beginning of TTI $t$, the SRA unit of the BS is required to derive the RB allocation set $N_{B}=\left\{N_{B 1}, \ldots, N_{B N_{M S}}\right\}$, where $N_{B u}$ is the number of RBs allocated to MS $u$, and the MCS allocation set $\mu=\left\{\mu_{1}, \ldots, \mu_{N_{M S}}\right\}$, where $\mu_{u}=\left\{\mu_{u, 1}, \ldots, \mu_{u, L_{u}}\right\}$ represents a set of MCSs assigned to each spatial stream $l$ of MS $u$, to effectively allocate RBs and MCSs, respectively. For simplicity, $t$ is dropped in our problem formulation presented hereafter. We formulate the SRA optimization problem to maximize the total utility $V$, i.e., the total weighted goodput, as follows: 


$$
\begin{aligned}
V=\max _{\mathcal{N}_{B}, \mu} \sum_{u=1}^{N_{M S}} \sum_{l=1}^{L_{u}} w_{u} r_{u, l}\left(\mathcal{N}_{B u}\right)\left[1-B L E R_{u, l}^{\left(\mu_{u, l}\right)}\left(\mathcal{N}_{B u}\right)\right] \\
\text { subject to } \mathcal{N}_{B k} \cap \mathcal{N}_{B j}=\varnothing \quad \forall k \neq j \\
\sum_{l=1}^{L_{u}} r_{u, l}\left(\mathcal{N}_{B u}\right) \leq Q_{u} \quad \forall(u, l) \\
B L E R_{u, l}^{\left(\mu_{u, l}\right)}\left(\mathcal{N}_{B u}\right) \leq B L E R_{0} \quad \forall(u, l)
\end{aligned}
$$

where $w_{u}$ is the weight of user $u$ and $B L E R_{u, l}^{\left(\mu_{u, l}\right)}$ is the Block Error Rate of user $u^{\prime}$ s spatial stream $l$ to which the MCS $\mu_{u, l}$ is assigned.

The SRA unit is required to maximize the utility function $V$ subject to these three constraints:

- An RB should be exclusively allocated to one user.

- The scheduler should allocate no more maximum transmission capacity than the number of bits in its queue to maintain the frugality constraint.

- The average $B L E R$ of $u$ does not exceed the upper bound, $B L E R_{0}$, for a minimum quality guarantee.

The proposed methodology in [13] uses an adaptive MCS [14] for each user $u$ considering his/her Channel State Information (CSI) and Queue State Information (QSI). Moreover, the authors proposed a greedy algorithm to allocate RBs to users efficiently. Essentially, it allocates the first RB to the user with the largest utility increase. It repeats this greedy approach until the set of non-allocated RBs becomes empty or there are no more active users to whom to allocate RBs. In this paper, we propose a cost-effective algorithm based on dynamic programming to allocate RBs optimally to active users, while applying the same adaptive MCS scheme used in [13].

\section{Dynamic Scheduling and Resource Allocation}

In general, a greedy algorithm makes a choice deemed best according to a certain criterion regardless of the choices it made before or will make in the future. Although it may find an effective solution in a reasonable time, it also results in a suboptimal solution when a series of local decisions fails to lead to a global optimum. The basic idea for dynamic programming is to solve subproblems optimally only once and store the results and look up the stored optimal solutions to the subproblems instead of recomputing them to compute the optimal solution for a given problem efficiently $[79,80]$.

In this paper, we design a new SRA algorithm by adapting the dynamic programming method for the 0/1 knapsack problem to optimize the utility of SRA for allocating RBs to active users with non-empty queues. It is challenging to design a cost-effective algorithm for $\mathrm{RB}$ allocation, since the 0/1 knapsack problem is NP-complete. In this section, we design a dynamic programming algorithm to maximize the utility defined in Section 3 in polynomial time and analyze the time complexity.

To this end, we first design the recursive structure of utility function $V$ to allocate free RBs, $N_{B}^{\text {free }}$, optimally to an arbitrary user $u$ where $Q_{u}>0$ as follows:

$$
V[u, k]= \begin{cases}\max \left(V[u-1, k], V[u-1, k-m[u]]+w[u] \times r_{u, l}(m[u])\right) & \text { if } m[u] \leq k \\ V[u-1, k] & \text { otherwise. }\end{cases}
$$

Here, $k$ is the number of the available RBs, $m[u]$ is the number of RBs required by the MS $u$ and $r_{u, l}(m[u])$ is the transmission capacity provided to MS $u$ by $m[u]$ RBs. If $m[u] \leq k$, MS $u$ can be assigned the required number of RBs. In this case, our dynamic programming method for SRA optimizes the total utility by assigning $m[u]$ RBs to MS $u$, if $V[u-1, k-m[u]]+w[u] \times r_{u, l}(m[u])>V[u-1, k]$ and updates the total utility as $V[u-1, k-m[u]]+w[u] \times r_{u, l}(m[u])$. Otherwise, it does not assign the RBs to MS $u$ and maintains the utility as $V[u-1, k]$. If $m[u]>k$; however, the RBs required by MS $u$ are 
unavailable; therefore, our approach cannot meet the requirement of MS $u$. As a result, the utility remains as $V[u-1, k]$. With this, we design the dynamic programming algorithm for SRA based on these recursive properties, as shown in Algorithm 1.

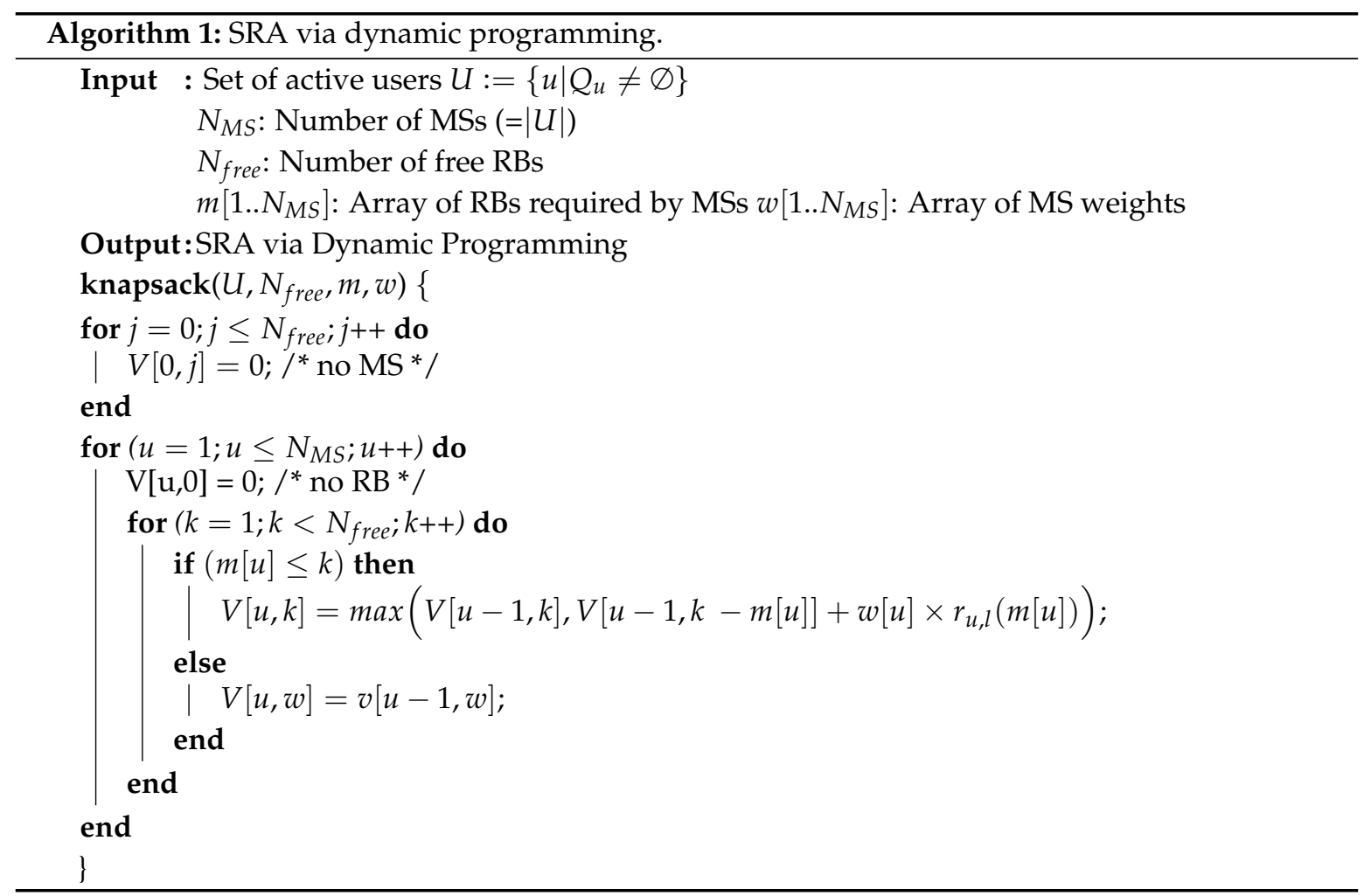

As discussed earlier, the base station has limited transmission capacity $r_{u, l}\left(t, N_{B_{u}}\right)$ at each TTI $t$, where the spatial stream, $l$, and number of RBs, $N_{B_{u}}$, represent the resources in the frequency and time domain, respectively. At the $5 \mathrm{G}$ base station, the user requests in a queue may be of eMBB, URLLC and eMTC types. As a result, it may demand various $m[u]$ RBs, such as 100, 25 and 6 for the eMBB, URLLC and eMTC use-cases, respectively. In this case, our utility function $V$ allocates the required RBs only if $m[u] \leq k$ and tries to maximize the transmission capacity $r_{u, l}$. If $m[u]>k$, it holds the request of user $u$ till the next TTI and schedules it later. Hence, the user requests of diverse use-cases at the $5 \mathrm{G}$ base station can be scheduled together by our SRA algorithm based on dynamic programming. By leveraging dynamic programming, we fully optimize the allocation of the transmission capacity at the base station at every TTI.

\section{Time Complexity Analysis}

The time complexity of Algorithm 1 is $O\left(N_{M S} \times N_{\text {all }}\right)$, where $N_{\text {all }}$ is the total number of RBs in a wireless communication frame at the BS. In general, when the number of items to consider is $n$ and the total capacity of the knapsack is $W$, the time complexity of the dynamic programming algorithm for the $0 / 1$ knapsack problem is $O(n W)$ [81]. $O(n W)$ is pseudo-polynomial complexity, since there is no guarantee that $W$ is a polynomial function of $n$, but it could be arbitrarily large (e.g., exponential with respect to $n)$. In practice, however, $N_{\text {all }}$ during a wireless communication frame is a fixed constant known a priori. For example, in LTE, one frame is $10 \mathrm{~ms}$, and $N_{\text {all }}$ is six and 100 when the channel bandwidth is $1.4 \mathrm{MHz}$ and $200 \mathrm{MHz}$, respectively. Each RB consists of 84 resource elements when each RB consists of seven symbols (time slots) in the time axis and 12 subcarriers (15 $\mathrm{kHz}$ each) in the frequency axis [82]. As long as $N_{\text {all }}$ remains a constant or is a polynomial function of $N_{M S}$ in practical implementations of the $5 \mathrm{G}$ standard, the time complexity of our algorithm remains polynomial. 


\section{Performance Evaluation}

We have compared the performance of the proposed SRA algorithm based on dynamic programming to that of the novel greedy algorithm [13], which is used as the baseline for performance comparisons in this paper. To evaluate the proposed SRA algorithm, we use the MATLAB LTE toolbox (version 2017a, Mathworks, Natick, MA, USA), with a 5G library that supports the system architecture as per the 3GPP recommendations [83], similar to the state-of-the-art work, such as $[13,14]$. For fair comparisons, we use the same simulation settings as the baseline [13]. To implement the greedy SRA and dynamic programming algorithms, we have modified the lteDLResourceGrid function.

Performance is measured in terms of goodput and fairness [84] for the two dominant 5G waveforms, i.e., OFDM and FBMC [85-87]. In the rest of this section, we call the proposed method dynamic and the baseline method [13] as greedy for brevity. The greedy-FBMC and greedy-OFDM conventions are used to address the reproduced baseline approach [13] for the FBMC and OFDM waveforms, respectively. Their results are plotted using dotted lines. Similarly, dynamic-FBMC and dynamic-OFDM refer to the proposed SRA algorithm with the FBMC and OFDM waveforms, respectively. The results of dynamic-FBMC and dynamic-OFDM are plotted with solid lines for clarity of presentation.

In our previous work [17], we evaluated performance for LTE with a 20-MHz bandwidth and $100 \mathrm{RBs}$, since the 5G standardization was still underway at that time. As the 5G standardization has been finalized, we have three broad categories of use cases: eMBB, URLLC and eMTC for 5G NSA, as discussed before. From the scheduling perspective, we can distinguish these use cases as different requirements for bandwidth and RBs: (1) eMBB with a 100-MHz bandwidth with $500 \mathrm{RBs}$ or more; (2) URLLC with up to $5 \mathrm{MHz}$ and $25 \mathrm{RBs}$; and (3) eMTC with $1.4 \mathrm{MHz}$ and $6 \mathrm{RBs}$ or less. Using these settings, we compare the performance of greedy-FBMC, greedy-OFDM, dynamic-FBMC and dynamic-OFDM. Unfortunately, during the performance evaluation, we found two limitations of the LTE toolbox: (1) for a single cell, only up to 16 users can be simulated; and (2) a maximum of 100 RBs can be modeled in the PHY layer; hence, we cannot evaluate the eMBB case in this paper. A more extensive evaluation is reserved for future work. The results of the URLLC and eMTC use-cases are shown in Figures 3 and 4 for goodput and in Figures 5 and 6 for fairness measurements. A detailed discussion of goodput and fairness measurements is given in the following subsections.

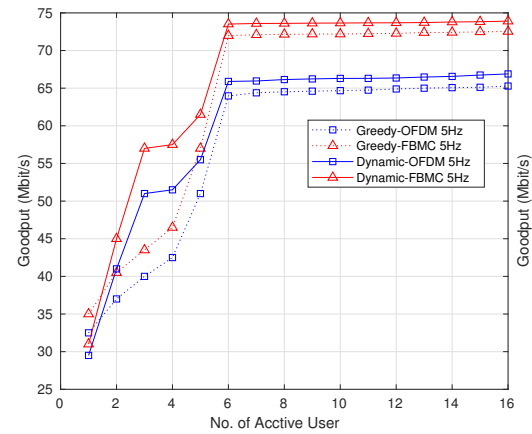

(a) EPA Channel for LTE

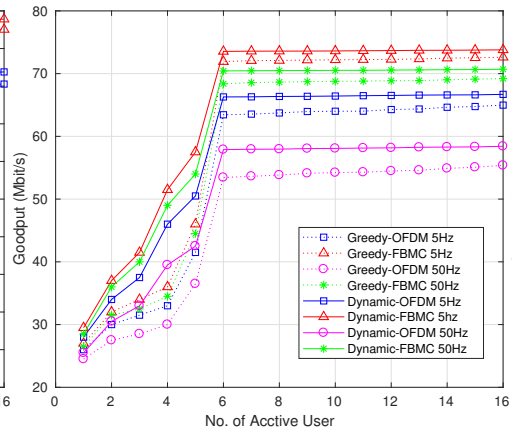

(b) EVA Channel for LTE

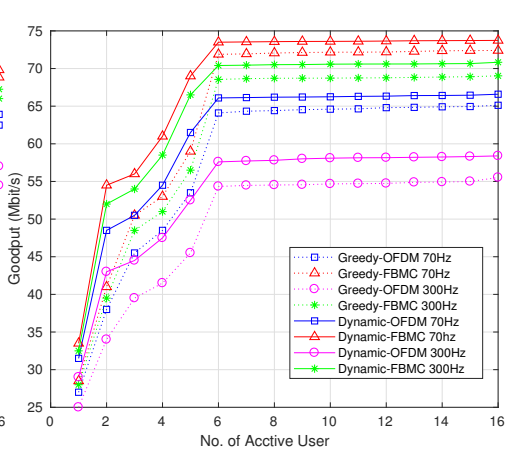

(c) ETU Channel for LTE

Figure 3. Cont. 


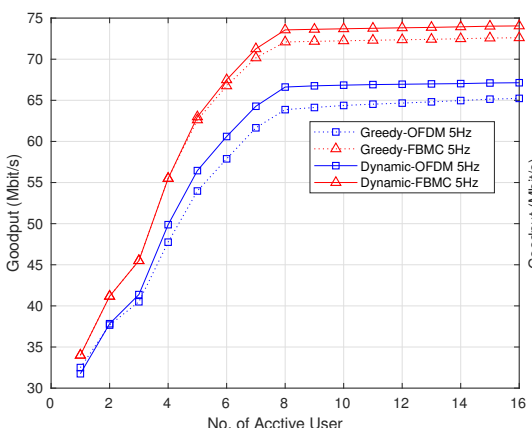

(d) EPA Channel for URLLC

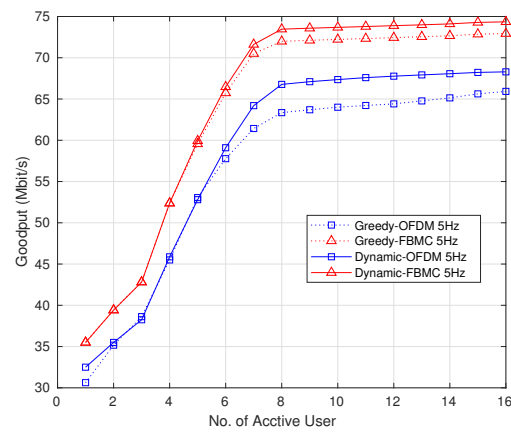

(g) EPA Channel for eMTC

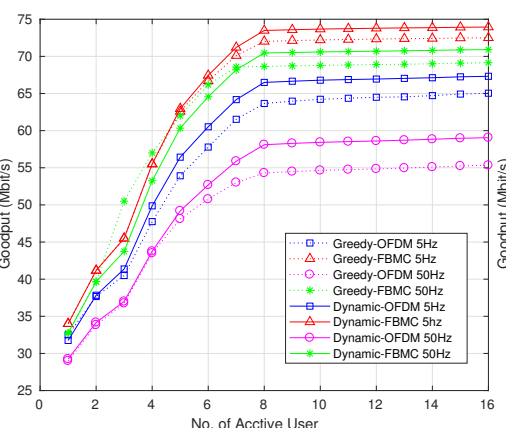

(e) EVA Channel for URLLC

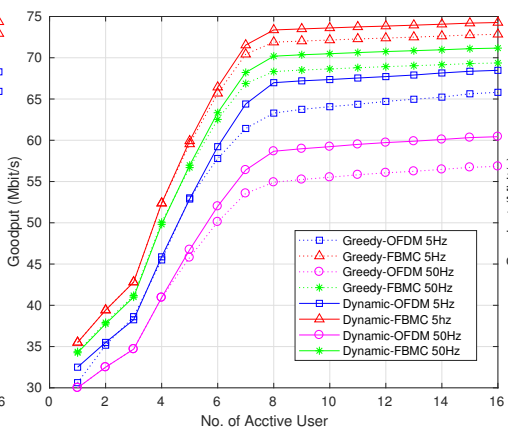

(h) EVA Channel for eMTC

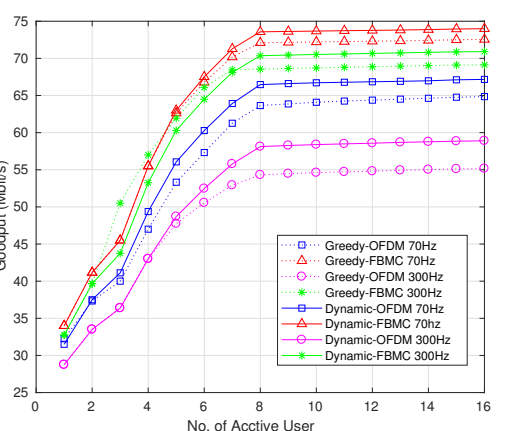

(f) ETU Channel for URLLC

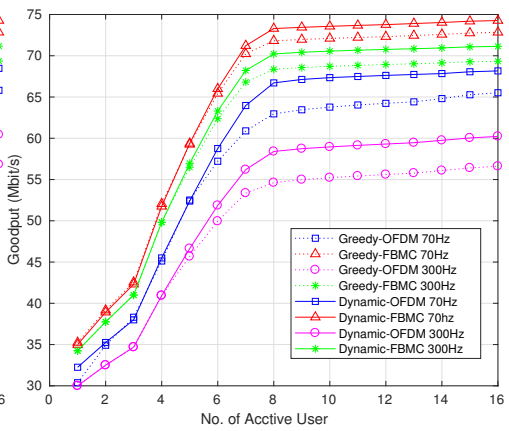

(i) ETU Channel for eMTC

Figure 3. Average goodput results for the greedy and dynamic approaches. EPA, Extended Pedestrian A; EVA, Extended Vehicular A; ETU, Extended Typical Urban; LTE, Long Term Evolution; URLLC, Ultra-Reliable Low Latency Communication; eMTC, enhanced Machine Type Communication.

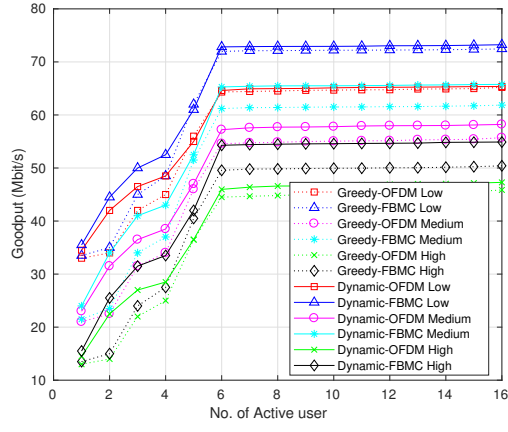

(a) EPA Channel for LTE

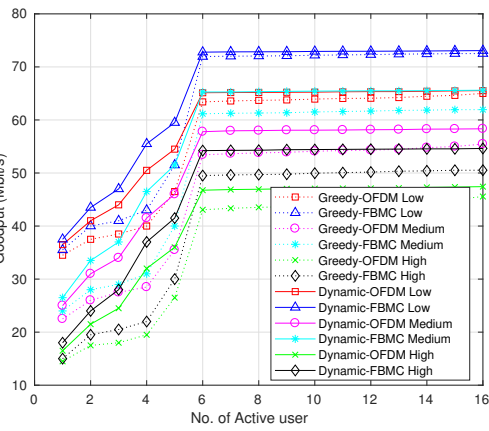

(b) EVA Channel for LTE

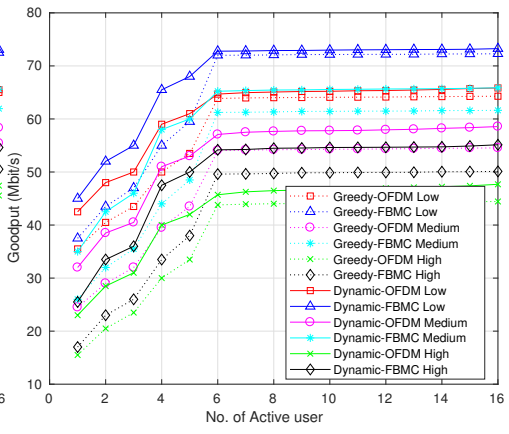

(c) ETU Channel for LTE

Figure 4. Cont. 


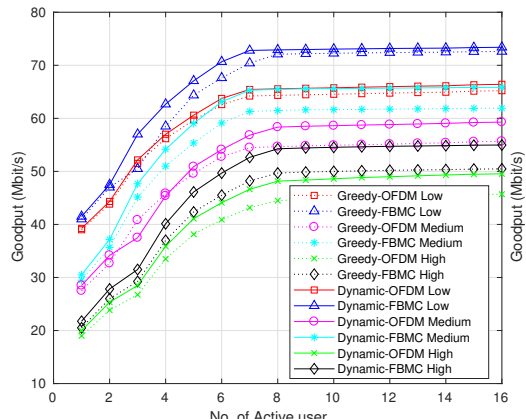

(d) EPA Channel for URLLC

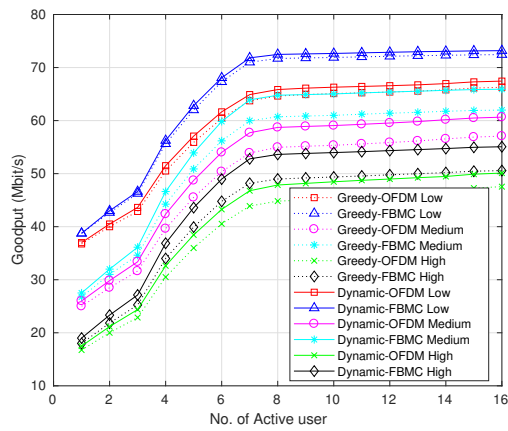

(g) EPA Channel for eMTC

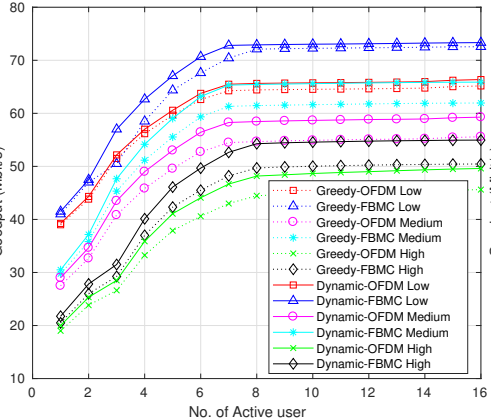

(e) EVA Channel for URLLC

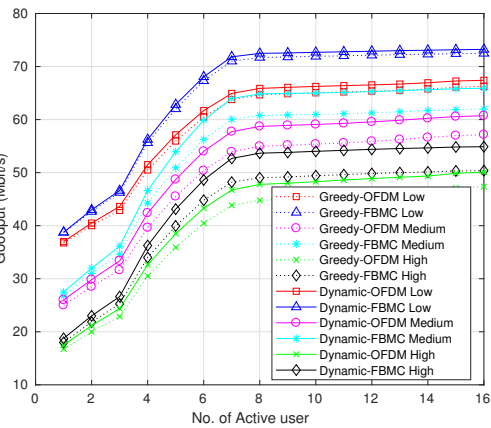

(h) EVA Channel for eMTC

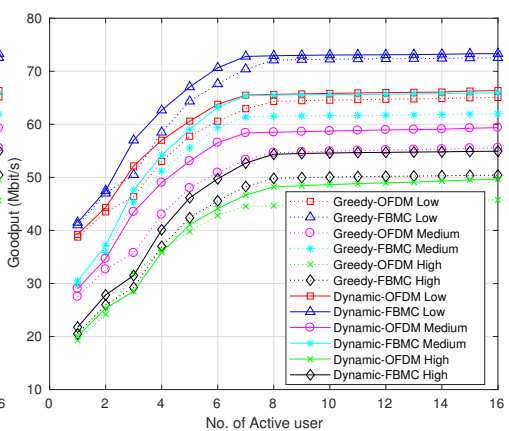

(f) ETU Channel for URLLC

Figure 4. Impact of correlations on average goodput for the greedy and dynamic approaches.

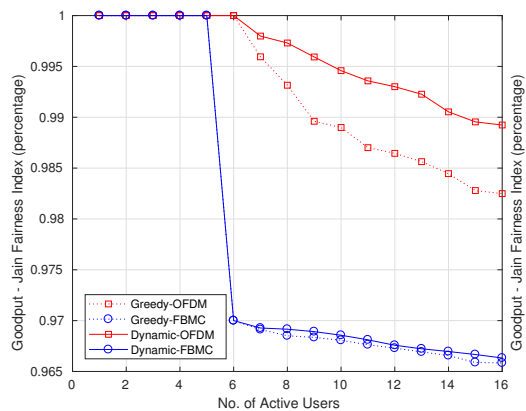

(a) EPA Channel for LTE

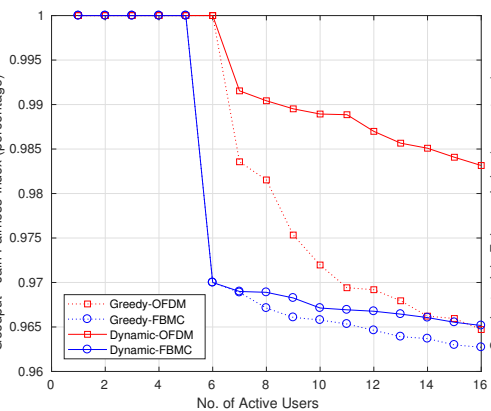

(b) EVA Channel for LTE

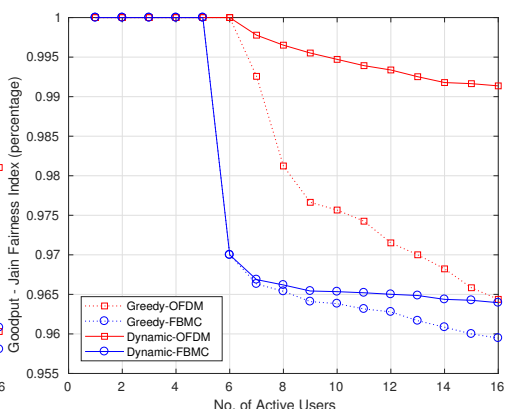

(c) ETU Channel for LTE

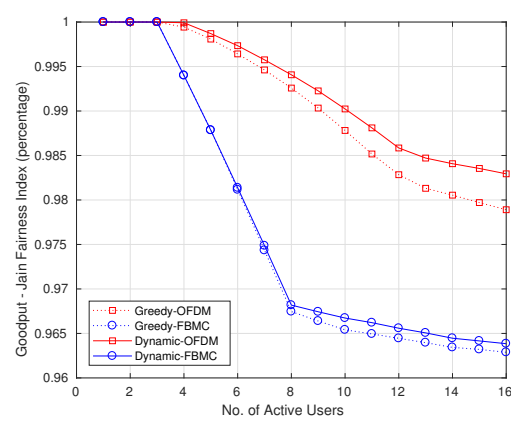

(d) EPA Channel for URLLC

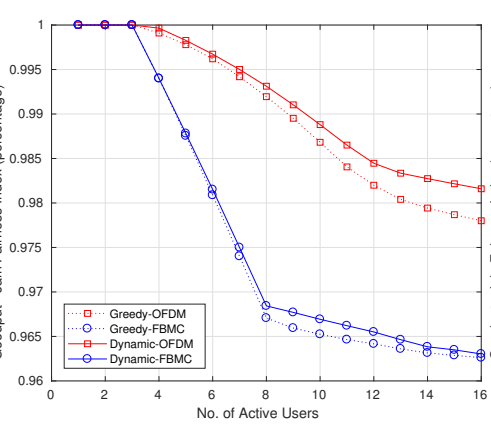

(e) EVA Channel for URLLC

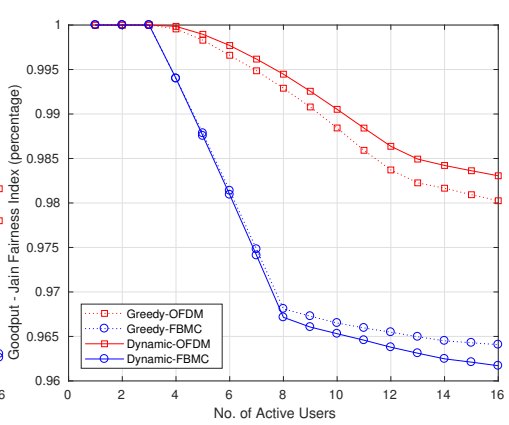

(f) ETU Channel for URLLC

Figure 5. Cont. 


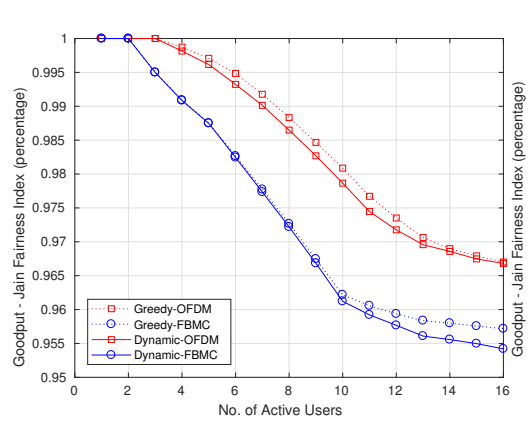

(d) EPA Channel for eMTC

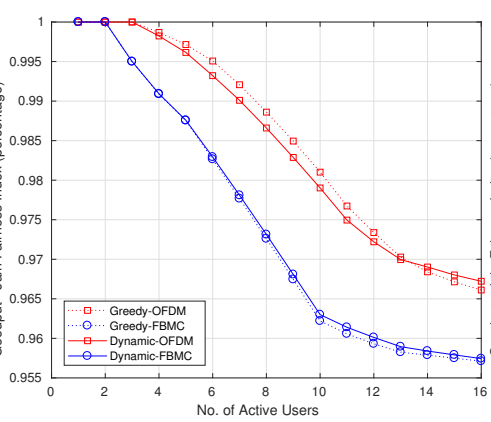

(e) EVA Channel for eMTC

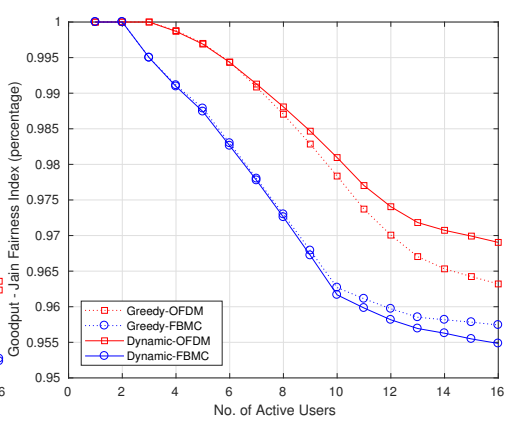

(f) ETU Channel for eMTC

Figure 5. Goodput Jain Fairness Index for the greedy and dynamic approaches.

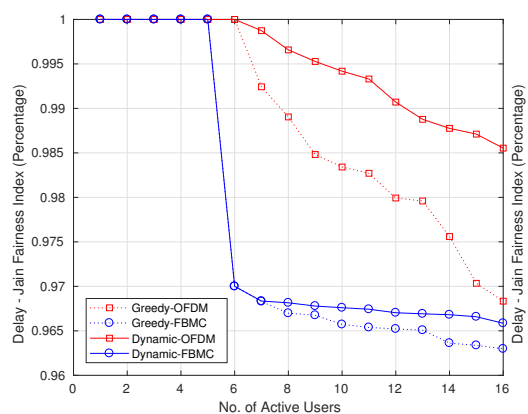

(g) EPA Channel for LTE

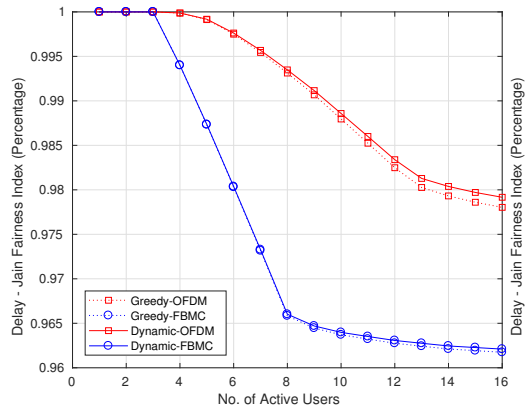

(j) EPA Channel for URLLC

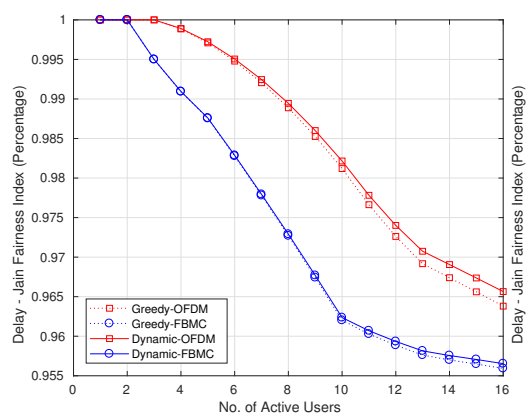

(m) EPA Channel for eMTC

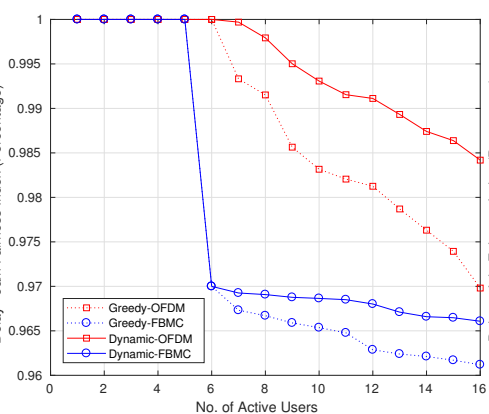

(h) EVA Channel for LTE

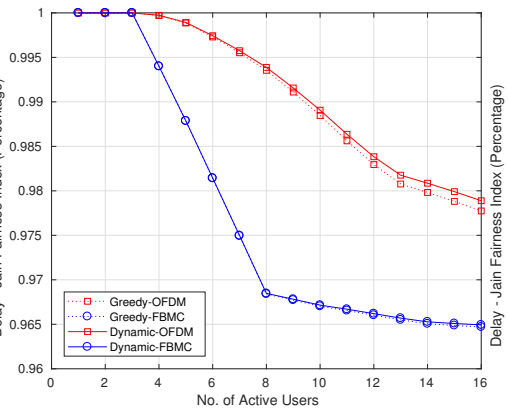

(k) EVA Channel for URLLC

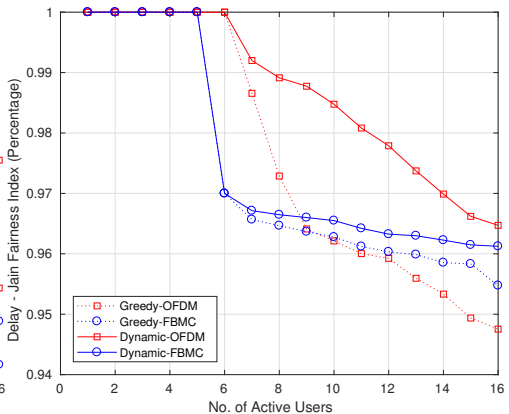

(i) ETU Channel for LTE

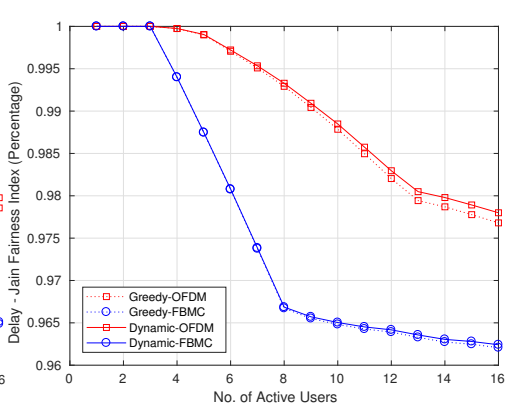

(1) ETU Channel for URLLC

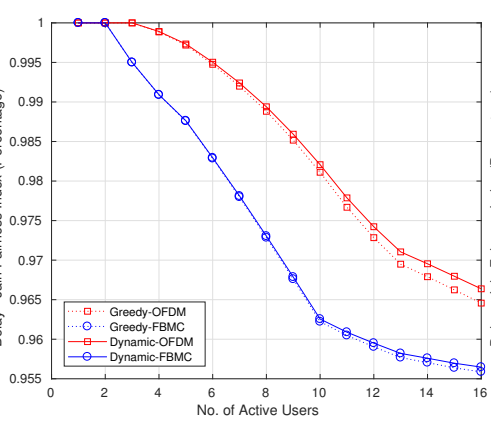

(n) EVA Channel for eMTC

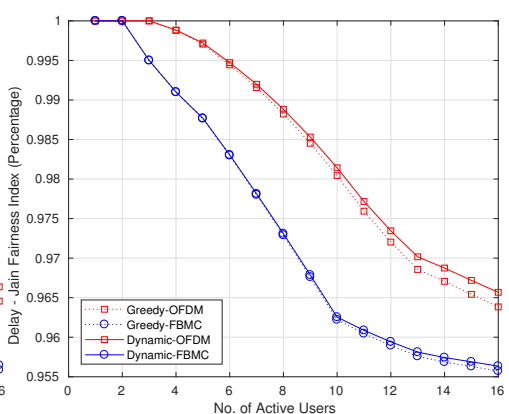

(o) ETU Channel for eMTC

Figure 6. Delay Jain Fairness Index for the greedy and dynamic approaches. 


\subsection{Goodput Measurements}

Goodput measurements are important at the application-level, since it shows the rate of successful data packet delivery observed on the UE side. Different from throughput, a goodput measurement excludes packet retransmissions; hence, the goodput can be comparatively lower than the throughput. However, in the case of noisy environments, it is important to measure the goodput to verify the successful delivery of packets received at a UE in a cell. To measure the goodput, we consider the delay and Doppler parameters as shown in Table 2.

As shown in Table 2, we consider three delay spread channel models: (1) the Extended Pedestrian A (EPA), (2) the Extended Vehicular A (EVA) and (3) the Extended Typical Urban (ETU) models. Each of these channel models has different delay profiles. EPA has the lowest delay and interference. EVA and ETU have higher delay and interference, in that order. We have also considered different correlation profiles as: (1) low, (2) medium and (3) high between the base station $\alpha$ and mobile station $\beta$, as shown in Table 2 .

Table 2. Channel model parameters [83].

\begin{tabular}{ccccccccc}
\hline & & \multicolumn{4}{c}{ Correlation Profiles } \\
\cline { 3 - 7 } Channel Model & Doppler Frequency (Hz) & Low & Medium & \multicolumn{3}{c}{ High } \\
\cline { 3 - 8 } & & $\boldsymbol{\alpha}$ & $\boldsymbol{\beta}$ & $\boldsymbol{\alpha}$ & $\boldsymbol{\beta}$ & $\boldsymbol{\alpha}$ & $\boldsymbol{\beta}$ \\
\hline EPA & 5 & 0 & 0 & 0.3 & 0.9 & 0.9 & 0.9 \\
EVA & 5,50 & 0 & 0 & 0.3 & 0.9 & 0.9 & 0.9 \\
ETU & 70,300 & 0 & 0 & 0.3 & 0.9 & 0.9 & 0.9 \\
\hline
\end{tabular}

\subsubsection{Average Goodput for Different Doppler Frequencies}

In a dense environment, signals may be reflected or the mobility of users may cause the Doppler effect in signals. The Doppler effect may reduce the overall performance of the network. Hence, in this paper, we have tested the proposed SRA and the baseline [13] against the standard Doppler frequencies for 5,50 and $300 \mathrm{~Hz}$, as suggested in [83]. Figure 3 shows the average goodput for different Doppler frequencies suggested for the EPA, EVA and ETU channel models. The performance is evaluated for the LTE, URLLC and eMTC use-cases.

The overall trend shows that the goodput increases as the number of users in a cell increases. It also shows that dynamic-FBMC outperforms dynamic-OFDM, greedy-FBMC and greedy-OFDM. For three users in a cell, dynamic-FBMC outperforms dynamic-OFDM, greedy-FBMC and greedy-OFDM by approximately $6 \mathrm{Mbps}(\approx 11.7 \%), 13 \mathrm{Mbps}(\approx 29.5 \%)$ and $17 \mathrm{Mbps}(\approx 42.5 \%)$, as shown in Figure 3a-c for the LTE use case. For URLLC and eMTC, its goodput is higher than the others' by up to approximately 1-3 Mbps, as shown in Figure $3 \mathrm{~d}-\mathrm{f}$ for URLLC and Figure $3 \mathrm{~g}-\mathrm{i}$ for eMTC. In the case of more than six users in a cell, dynamic-FBMC outperforms greedy-FBMC by up to 1-3 Mbps $(\approx 1.3-5.4 \%)$ and dynamic-OFDM by up to $10 \mathrm{Mbps}(\approx 17.24 \%)$ with different Doppler frequencies, as shown in Figure $3 \mathrm{~d}-\mathrm{f}$ for URLLC and Figure $3 \mathrm{~g}-\mathrm{i}$ for eMTC. Similarly, dynamic-OFDM outperforms greedy-OFDM by $2-3$ Mbps ( $\approx 5.4 \%)$ for the EPA, EVA and ETU channels with different Doppler frequencies, as shown in Figure 3. Overall, dynamic-FBMC outperforms dynamic-OFDM by up to $8-10 \mathrm{Mbps}$ ( $\approx 12.12-17.24 \%$ ) for different Doppler frequencies, as shown in Figure 3a-c for LTE, Figure $3 \mathrm{~d}-\mathrm{f}$ for URLLC and Figure $3 \mathrm{~g}-\mathrm{i}$ for eMTC when more than six users are present in the cell.

\subsubsection{Impact of Correlation on Average Goodput}

The incoming signal can be correlated with the transmitting and receiving antennas. The higher correlation of antennas leads to degradation of the overall system performance. As 5G uses MIMO technology, it is important to measure the correlation. In this paper, we have considered the standard high, low and medium correlation profiles, as shown in Table 2, where $\alpha$ means the base station and $\beta$ 
means the UE side correlation, as per [83]. The results are shown for the LTE, URLLC and eMTC use cases in Figure 4 with EPA, EVA and ETU channel models and low, medium and high correlation.

The overall trends show that the proposed dynamic approach achieves similar performance to that of the baseline for the low correlation profile as plotted in Figure $4 \mathrm{a}-\mathrm{c}$ for LTE, Figure $4 \mathrm{~d}-\mathrm{f}$ for URLLC and Figure 4g-i for eMTC. When there are less than six users in a cell with respect to the LTE use case, dynamic improves the goodput by approximately 8-10 Mbps $(\approx 11.1-18.1 \%)$, as shown in Figure $4 \mathrm{a}-\mathrm{c}$. The biggest goodput enhancement is approximately $14 \mathrm{Mbps}(\approx 60 \%)$, achieved by dynamic-FBMC-high over greedy-FBMC-high (the black solid and dotted curves in Figure $4 a)$. The dynamic-FBMC outperforms the greedy-FBMC by up to $4 \mathrm{Mbps}(\approx 7.27 \%)$ in the case of medium correlation and up to $5 \mathrm{Mbps}(\approx 9.09 \%)$ for high correlation profiles, as shown in Figure $4 \mathrm{~d}-\mathrm{f}$ for URLLC and Figure $4 \mathrm{~g}-\mathrm{i}$ for eMTC, respectively. Overall, dynamic-FBMC outperforms dynamic-OFDM by up to $8 \mathrm{Mbps}(\approx 16 \%)$, as shown in Figure $4 \mathrm{~d}-\mathrm{f}$ for URLLC and Figure $4 \mathrm{~g}-\mathrm{i}$ for eMTC, respectively.

\subsection{Fairness Measurements}

Fairness is a measure to calculate how fairly the base station is allocating resources to the requests made by multiple users. In the case of heavy traffic, a user request may have to wait longer to get resources and may even starve. Hence, a fairness analysis is important for any scheduling scheme. To ensure the fairness of our proposed SRA algorithm, we have used Jain's fairness measure. Jain et al. [84] proposed a methodology to calculate the fairness index among the users. If the measured goodput of the users is $T=\left\{T_{1}, \ldots, T_{n}\right\}$ and the required fair goodput is $O=\left\{O_{1}, \ldots, O_{n}\right\}$, then the normalized goodput is $X_{n}=\frac{T_{n}}{O_{n}}$. The overall goodput fairness is measured as follows:

$$
\text { FairnessIndex }=\frac{\left(\sum X_{n}^{2}\right)}{n \sum X_{n}^{2}}
$$

The delay fairness can be derived similarly from the above equation. Figures 5 and 6 show the results of fairness analysis in terms of average goodput and delay. The fairness is bounded between zero and one (i.e., 100\%). If Jain Fairness Index equals to 1, perfect fairness is achieved. On the other hand, zero fairness means starvation. There are more general fairness indicators $[88,89]$. In this paper, however, our main objective is to compare the performance of our approach to that of the advanced greedy baseline [13]. For fair comparisons, we use the same fairness metric and simulation settings as [13]. Using the more general fairness indicators and using other waveforms are reserved for future work.

In this paper, we consider two dominant 5G waveforms, OFDM and FBMC, as discussed before. A major implementation comparison between them is the number of complex symbol multiplications. In OFDM, the number of multiplications per symbol that the split-radix algorithm has is:

$$
C_{F F T / I F F T}=M((\log (M)-3)+4)
$$

where $M$ is the number of complex symbols. FFT and IFFT stand for the FFT and IFFT on the receiver and transmitter side, respectively.

In FBMC, the number of multiplications per symbol is:

$$
C_{S F B / A F B}=2 M((\log (M)-3)+4 K)
$$

where the $S F B$ and $A F B$ are the Synthesis and Analysis Filter Banks at the transmitter and receiver side, respectively.

OFDM suffers from poor spectral selectivity, which leads to marginal degradation in fairness as the number of users increases, as plotted in Figures 5 and 6. FBMC has higher selectivity, but requires more symbol multiplications. 


\subsubsection{Goodput Fairness}

Goodput fairness measures how fairly the scheduler allocates the bandwidth. Figure 5 shows the goodput fairness for LTE, URLLC and eMTC use-cases with the EPA, EVA and ETU channel. The overall results show that the JFI index of the FBMC waveform is slightly lower than that of the OFDM due to its complex nature. For all these cases, the proposed SRA and baseline approaches show full fairness $(100 \%)$ for up to three users. In the case of the URLLC and eMTC use-cases, dynamic and greedy show similar performance for OFDM and FBMC waveforms, as shown in Figure $5 d-i$. In the case of LTE and URLLC, the JFI index remains more than $96 \%$, and for eMTC, it is more than $95 \%$, as shown in Figure 5. Dynamic-OFDM outperforms dynamic-FBMC by up to $0.02 \%(\approx 2.5 \%)$, as shown in Figure $5 \mathrm{a}-\mathrm{c}$ for LTE and in Figure $5 \mathrm{~d}-\mathrm{f}$ for URLLC. In the case of eMTC, dynamic-OFDM outperforms dynamic-FBMC by up to $0.01 \%(\approx 1.5 \%)$, as shown in Figure $5 \mathrm{~g}-\mathrm{i}$.

\subsubsection{Delay Fairness}

Delay fairness measures how long an arbitrary user needs to wait for resource allocation. Figure 6 plots the delay fairness for LTE, URLLC and eMTC with the EPA, EVA and ETU channel models. For all these cases, the proposed SRA and baseline approaches show full fairness (100\%) for up to three users. In the case of the URLLC and eMTC use-cases, dynamic and greedy show similar performance for OFDM and FBMC waveforms, as shown in Figure 6d-i. Dynamic-OFDM outperforms dynamic-FBMC by up to $0.01 \%(\approx 1.5 \%)$, as shown in Figure $6 \mathrm{a}-\mathrm{c}$ for LTE and Figure $6 \mathrm{~d}-\mathrm{f}$ for URLLC. In the case of eMTC, dynamic-OFDM outperforms dynamic-FBMC by up to $0.01 \%(\approx 1 \%)$, as shown in Figure $6 \mathrm{~g}-\mathrm{i}$. In the other cases, its fairness is slightly higher than or similar to the fairness of greedy.

\section{Conclusions and Future Work}

The upcoming 5G technology is envisioned to support diverse use-cases such as enhanced Mobile Broadband (eMBB), enhanced Machine Type Communication (eMTC), and Ultra-Reliable Low Latency Communication (URLLC). These use cases may generate different types of traffic at the base station. The first generation of 5G comes with backward LTE compatibility, which generates even more diversity in traffic at the base station. Hence, we need an effective Scheduling and Resource Allocation (SRA) at the 5G base station. In this paper, we have proposed a new SRA algorithm based on dynamic programming that can accommodate diverse use-cases of $5 \mathrm{G}$ and make effective SRA decisions. The simulation results show the robust performance of the proposed SRA for the two dominant waveforms for 5G, i.e., Orthogonal Frequency Multiplexing (OFDM) and Filter Bank Multi-Carrier (FBMC). For LTE, our SRA method outperforms the advanced greedy SRA algorithm [13] by up to approximately $60 \%, 2.6 \%$ and $1.6 \%$ in terms of goodput, goodput fairness and delay fairness, as observed in [17]. In the case of URLLC and eMTC, our SRA algorithm continues to outperform [13] by up to $17.24 \%, 18.1 \%, 2.5 \%$ and $1.5 \%$ in terms of average goodput, correlation impact, goodput fairness and delay fairness. In the future, we will explore other $5 \mathrm{G} \mathrm{New}$ Radio (NR) waveforms and leverage them in our SRA framework. We will also investigate if the dynamic programming algorithm for SRA can be further extended to support V2X communication, which is significantly more challenging due to high-speed mobile stations.

Author Contributions: A.V. has developed the dynamic programming algorithm for 5G downlink scheduling and resource allocation. A.V. also performed the simulation study. K.-D.K. helped A.V. to design the algorithm and discussed the results with A.V. K.-D.K. also helped A.V. to write this paper.

Funding: This work was supported, in part, by NSF Grant CNS1526932.

Acknowledgments: We appreciate the guest editor and anonymous reviewers for their help to improve this paper.

Conflicts of Interest: The authors declare no conflict of interest. 


\section{References}

1. Internet of Things-number of connected devices worldwide 2012-2025 (Statista). Available online: https:/ / www.statista.com/statistics/471264/iot-number-of-connected-devices-worldwide/ (accessed on 14 November 2018).

2. Vestberg, H. CEO to Shareholders: 50 Billion Connections 2020; Technical Report; Ericsson: Stockholm, Sweden, 2010.

3. Internet of Things forecast_Ericsson. Available online: https:/ /www.ericsson.com/en/mobility-report/ internet-of-things-forecast (accessed on 14 November 2018).

4. Liu, G.; Jiang, D. 5G: Vision and requirements for mobile communication system towards year 2020. Chin. J. Eng. 2016, 2016, 5974586. [CrossRef]

5. Akpakwu, G.A.; Silva, B.J.; Hancke, G.P.; Abu-Mahfouz, A.M. A survey on 5G networks for the Internet of things: Communication technologies and challenges. IEEE Access 2018, 6, 3619-3647. [CrossRef]

6. Gupta, A.; Jha, R.K. A survey of 5G network: Architecture and emerging technologies. IEEE Access 2015, 3, 1206-1232. [CrossRef]

7. Agiwal, M.; Roy, A.; Saxena, N. Next generation 5G wireless networks: A comprehensive survey. IEEE Commun. Surv. Tutor. 2016, 18, 1617-1655. [CrossRef]

8. Recommendation ITU-R M.2083-0 Policy on Intellectual Property Right (IPR) Series of ITU-R Recommendations. Available online: https:/ / www.itu.int/dms_pubrec/itu-r/rec/m/R-REC-M.2083-0201509-I!!PDF-E.pdf (accessed on 14 November 2018).

9. CISCO. 5G Non Standalone Solution Overview; Technical Report; CISCO: San Jose, CA, USA, 2018.

10. Mediatek. 5G: What Is Standalone Vs Non-Standalone Networks? Technical Report; Mediatek: Taiwan, 2018.

11. Giordani, M.; Polese, M.; Roy, A.; Castor, D.; Zorzi, M. Standalone and Non-Standalone Beam Management for 3GPP NR at mmWaves. arXiv 2018, arXiv:1805.04268.

12. Ghosh, A. 5G new radio design. In Proceedings of the IEEE 86th Vehicular Technology Conference: VTC2017-Fall, Toronto, Canada, 24-27 September 2017.

13. Femenias, G.; Riera-Palou, F.; Mestre, X.; Olmos, J.J. Downlink Scheduling and Resource Allocation for 5G MIMO-Multicarrier: OFDM vs FBMC/OQAM. IEEE Access 2017, 5, 13770-13786. [CrossRef]

14. Femenias, G.; Riera-Palou, F. Scheduling and resource allocation in downlink multiuser MIMO-OFDMA systems. IEEE Trans. Commun. 2016, 64, 2019-2034. [CrossRef]

15. Cisco Global Cloud Index: Forecast and Methodology, 2015-2020. Available online: https:/ /www.iotjournaal. nl/wp-content/uploads/2017/02/white-paper-c11-738085.pdf (accessed on 14 November 2018).

16. Dañobeitia, B.; Femenias, G.; Riera-Palou, F. Unified approach to cross-layer scheduling and resource allocation in OFDMA wireless networks. EURASIP J. Wirel. Commun. Netw. 2012, 2012, 145. [CrossRef]

17. Vora, A.; Kang, K.D. Downlink Scheduling and Resource Allocation for 5G MIMO Multicarrier Systems. In Proceedings of the 2018 IEEE 5G World Forum (5GWF), Santa Clara, CA, USA, 9-11 July 2018.

18. Marincic, A.; Simunic, D. Performance evaluation of different scheduling algorithms in LTE systems. In Proceedings of the 39th International Convention on Information and Communication Technology, Electronics and Microelectronics (MIPRO), Opatija, Croatia, 30 May-3 June 2016; pp. 595-600.

19. Jabbar, A.I.A.; Abdullah, F.Y. Long term evolution scheduling algorithms in wireless sensor networks. Int. J. Comput. Appl. 2015, 121, 12-16.

20. Trivedi, R.D.; Patel, M.C. Comparison of Different Scheduling Algorithm for LTE. Int. J. Emerg. Technol. Adv. 2014, 4, 334-339.

21. Thakkar, A. Downlink Resource Allocation in LTE, 2011. Available online: https:/ /github.com/adthakkar/ LTE_Downlink_Survey/tree/master/paper (accessed on 14 November 2018).

22. Atef, I.; Sourour, E. Modified proportional fair for LTE femto cells with eICIC. In Proceedings of the 4th International Conference on Wireless Communications, Vehicular Technology, Information Theory and Aerospace \& Electronic Systems (VITAE), Aalborg, Denmark, 11-14 May 2014; pp. 1-5.

23. Dhameliya, N.; Bhoomarker, R.; Zafar, S. Maximization of throughput with advanced proportional fair algorithm for LTE-advanced. In Proceedings of the 2014 IEEE Students' Conference on Electrical, Electronics and Computer Science, Bhopal, India, 1-2 March 2014; pp. 1-4.

24. Muller, M.K.; Schwarz, S.; Rupp, M. QoS investigation of proportional fair scheduling in LTE networks. In Proceedings of the 2013 IEEE Wireless Days Conference, Valencia, Spain, 13-15 November 2013; pp. 1-4. 
25. Zubairi, J.A.; Erdogan, E.; Reich, S. Experiments in fair scheduling in 4G WiMAX and LTE. In Proceedings of the 2015 IEEE International Conference on High Performance Computing \& Simulation (HPCS), Amsterdam, The Netherlands, 20-24 July 2015; pp. 277-282.

26. Deng, R.; He, J.; Chen, Q.; Chen, M.; Liu, Y.; Liu, J.; Chen, L. A serial IFFT precoding scheme to mitigate the periodic noise in OFDM System. IEEE Commun. Lett. 2016, 20, 1301-1304. [CrossRef]

27. Deng, H.; Wang, Y.; Wu, C. Cognitive radio: A method to achieve spectrum sharing in LTE-R system. In Proceedings of the 2018 IEEE Network Operations and Management Symposium, Taipei, Taiwan, 23-27 April 2018; pp. 1-5.

28. Zhou, W.; Chen, W.; Tan, Z.; Chen, S.; Zhang, Y. A modified RR scheduling scheme based CoMP in LTE-A system. In Proceedings of the 2011 IET International Conference on Communication Technology and Application, Beijing, China, 14-16 October 2011; pp. 176-180.

29. Yang, D.; Bastos, J.; Verikoukis, C.; Rodriguez, J. Location-aided round robin scheduling for fractional frequency reused LTE-A relay network. In Proceedings of the 2012 IEEE International Workshop on Computer Aided Modeling and Design of Communication Links and Networks, Barcelona, Spain, 17-19 September 2012; pp. 11-15.

30. Damera, B.; Babu, P.C.; Mohamed, J.S. Optimized MCE scheduling algorithm to allocate radio resources using evolved Round Robin scheduling. In Proceedings of the 2016 IEEE International Conference on Applied and Theoretical Computing and Communication Technology, Bangalore, India, 21-23 July 2016; pp. 770-775.

31. Nguyen, D.H.; Nguyen, H.; Renault, E. Performance evaluation of E-MQS scheduler with Mobility in LTE heterogeneous network. In Proceedings of the 2017 IEEE International Conference on Communications, Paris, France, 21-25 May 2017; pp. 1-6.

32. Suganya, S.; Maheshwari, S.; Latha, Y.S.; Ramesh, C. Resource scheduling algorithms for LTE using weights. In Proceedings of the 2016 IEEE International Conference on Applied and Theoretical Computing and Communication Technology, Bangalore, India, 21-23 July 2016; pp. 264-269.

33. Grondalen, O.; Zanella, A.; Mahmood, K.; Carpin, M.; Rasool, J.; Osterbo, O.N. Scheduling policies in time and frequency domains for LTE downlink channel: A performance comparison. IEEE Trans. Veh. Technol. 2017, 66, 3345-3360. [CrossRef]

34. Chen, S.; Yao, W.; Hanzo, L. Semi-Blind Adaptive Spatial Equalization for MIMO Systems with High-Order QAM Signalling. IEEE Trans. Wirel. Commun. 2008, 7, 4486-4491. [CrossRef]

35. Sudheep, S.; Rebekka, B. Proportional equal throughput scheduler-A very fair scheduling approach in LTE downlink. In Proceedings of the 2014 IEEE International Conference on Information Communication and Embedded Systems (ICICES2014), Chennai, India, 27-28 Febuary 2014; pp. 1-6.

36. Liu, Y.; Huynh, M.; Ghosal, D. Enhanced DRX-aware scheduling for mobile users in LTE networks. In Proceedings of the 2016 IEEE International Conference on Computing, Networking and Communications, Kauai, HI, USA, 15-18 Febuary 2016; pp. 1-5.

37. Nasralla, M.M.; Martini, M.G. A downlink scheduling approach for balancing QoS in LTE wireless networks. In Proceedings of the 2013 IEEE Annual International Symposium on Personal, Indoor, and Mobile Radio Communications, London, UK, 8-11 September 2013; pp. 1571-1575.

38. Lai, W.P.; Liou, E.C.; Chen, P.C. Radio resource scheduling using packet-level service differentiation for video over the LTE downlink. In Proceedings of the 2014 IEEE International Conference on Communications in China, Shanghai, China, 13-15 October 2014; pp. 851-855.

39. Vizzarri, A. Analysis of VoIP over LTE end-to-end performances in congested scenarios. In Proceedings of the 2014 IEEE 2nd International Conference on Artificial Intelligence, Modelling and Simulation, Madrid, Spain, 18-20 November 2014; pp. 339-343.

40. Choi, M.T.; Kim, J.H. A design of real-time traffic sensitive CSAT access control frame for unlicensed LTE operations. In Proceedings of the 2016 IEEE International Conference on Information and Communication Technology Convergence, Jeju, Korea, 19-21 October 2016; pp. 982-985.

41. Choi, S.; Jun, K.; Shin, Y.; Kang, S.; Choi, B. MAC scheduling scheme for VoIP traffic service in 3G LTE. In Proceedings of the 2007 IEEE Vehicular Technology Conference, Baltimore, MD, USA, 30 September3 October 2007; pp. 1441-1445.

42. Zhang, L.; Okamawari, T.; Fujii, T. Experimental analysis of TCP and UDP during LTE handover. In Proceedings of the 2012 IEEE Vehicular Technology Conference, Yokohama, Japan, 6-9 May 2012; pp. 1-5. 
43. Nagai, Y.; Zhang, L.; Okamawari, T.; Fujii, T. Delay performance analysis of LTE in various traffic patterns and radio propagation environments. In Proceedings of the 2013 IEEE Vehicular Technology Conference, Dresden, Germany, 2-5 June 2013; pp. 1-5.

44. Ognenoski, O.; Nasralla, M.M.; Razaak, M.; Martini, M.; Amon, P. DASH-based video transmission over LTE networks. In Proceedings of the 2015 IEEE International Conference on Communication Workshop (ICCW), London, UK, 8-12 June 2015; pp. 1783-1787.

45. Savona, G.; Debono, C.J. Schedulers for MPEG-DASH compliant multi-view video over LTE. In Proceedings of 2016 IEEE the International Conference on Telecommunications and Multimedia, Heraklion, Greece, 25-27 July 2016; pp. 1-6.

46. Gao, S.; Tao, M. Joint multicast scheduling and user association for DASH-based video streaming over heterogeneous cellular networks. In Proceedings of the 2016 IEEE International Conference on Communications in China, Chengdu, China, 27-29 July 2016; pp. 1-6.

47. Zhao, M.; Gong, X.; Liang, J.; Wang, W.; Que, X.; Cheng, S. Scheduling and resource allocation for wireless dynamic adaptive streaming of scalable videos over HTTP. In Proceedings of the 2014 IEEE International Conference on Communications, Sydney, Australia, 10-14 June 2014; pp. 1681-1686.

48. Giluka, M.K.; Rajoria, N.; Kulkarni, A.C.; Sathya, V.; Tamma, B.R. Class based dynamic priority scheduling for uplink to support M2M communications in LTE. In Proceedings of the 2014 IEEE World Forum on Internet of Things, Seoul, Korea, 6-8 March 2014; pp. 313-317.

49. Khan, N.; Martini, M.G. QoE-based video delivery over LTE hierarchical architecture. In Proceedings of the 2016 IEEE Annual International Symposium on Personal, Indoor, and Mobile Radio Communications, Valencia, Spain, 4-8 September 2016; pp. 1-6.

50. Gong, Y.; Yan, B.; Lin, S.; Li, Y.; Guan, L. Priority-based LTE down-link packet scheduling for Smart Grid communication. In Proceedings of the 2016 IEEE International Conference on Computer and Communications, Chengdu, China, 14-17 October 2016; pp. 2308-2312.

51. Mostafa, A.E.; Gadallah, Y. A statistical priority-based scheduling metric for M2M communications in LTE networks. IEEE Access 2017, 5, 8106-8117. [CrossRef]

52. Gemici, O.F.; Hokelek, I.; Cirpan, H.A. Trade-off analysis of QoS-aware configurable LTE downlink schedulers. In Proceedings of the ICT 2013, Casablanca, Morocco, 6-8 May 2013; pp. 1-5.

53. Piro, G.; Grieco, L.; Boggia, G.; Camarda, P. QoS provisioning in LTE-A networks with relay nodes. In Proceedings of the 2012 IEEE IFIP Wireless Days, Dublin, Ireland, 21-23 November 2012; pp. 1-3.

54. Yusoff, R.; Baba, M.D.; Ali, D. Energy-efficient resource allocation scheduler with QoS aware supports for green LTE network. In Proceedings of the 2015 IEEE Control and System Graduate Research Colloquium, Shah Alam, Malaysia, 10-11 August 2015; pp. 109-111.

55. Abdalla, I.; Venkatesan, S. A QoE preserving M2M-aware hybrid scheduler for LTE uplink. In Proceedings of the 2013 IEEE International Conference on Selected Topics in Mobile and Wireless Networking, Montreal, QC, Canada, 19-21 August 2013; pp. 127-132.

56. Barakat, B.; Arshad, K. An adaptive hybrid scheduling algorithm for LTE-Advanced. In Proceedings of the 2015 IEEE International Conference on Telecommunications, Sydney, Australia, 27-29 April 2015; pp. 91-95.

57. Ramli, H.A.M. Performance of maximum-largest weighted delay first algorithm in long term evolution-advanced with carrier aggregation. In Proceedings of the 2014 IEEE Wireless Communications and Networking Conference, Istanbul, Turkey, 6-9 April 2014; pp. 1415-1420.

58. Chen, J.; Yang, W.; Gao, S.; Zhou, L. Scheduling algorithm with delay-limited for VoIP in LTE. In Proceedings of the 2015 IEEE Asia-Pacific Signal and Information Processing Association Annual Summit and Conference, Hong Kong, China, 16-19 December 2015; pp. 1-10.

59. Zakharov, Y.; Zheng, D. Weighted LS multiuser channel estimation for LTE. In Proceedings of the 2015 IEEE International Workshop on Signal Processing Advances in Wireless Communications, Stockholm, Sweden, 28 June-1 July 2015; pp. 405-409.

60. Hong, T.C.; Kang, K.; Ku, B.J.; Ahn, D.S. A scheduling algorithm for selective repeat HARQ type II in the mobile satellite system. In Proceedings of the 2013 IEEE International Conference on ICT Convergence, Jeju, Korea, 14-16 October 2013; pp. 902-906.

61. Ramli, H.A.M.; Sandrasegaran, K.; Basukala, R.; Afrin, T.S. HARQ aware scheduling algorithm for the downlink LTE system. In Proceedings of the 2011 IEEE Fourth International Conference on Modeling, Simulation and Applied Optimization, Kuala Lumpur, Malaysia, 19-21 April 2011; pp. 1-4. 
62. Kim, M.; Kim, S.; Lim, Y. An implementation of downlink asynchronous HARQ for LTE TDD system. In Proceedings of the 2012 IEEE Radio and Wireless Symposium, Santa Clara, CA, USA, 15-18 January 2012; pp. 271-274.

63. Ang, E.M.; Wee, K.K.; Pang, Y.H.; Lau, S.H. Two-level scheduling framework with frame level scheduling and exponential rule in wireless network. In Proceedings of the 2014 IEEE International Conference on Information Science \& Applications, Seoul, Korea, 6-9 May 2014; pp. 1-4.

64. Afroz, F.; Sandrasegaran, K.; Ghosal, P. Performance analysis of PF, M-LWDF and EXP/PF packet scheduling algorithms in 3GPP LTE downlink. In Proceedings of the 2014 IEEE Australasian Telecommunication Networks and Applications Conference, Southbank, Australia, 26-28 November 2014; pp. 87-92.

65. Trabelsi, S.; Belghith, A.; Zarai, F.; Obaidat, M.S. Performance evaluation of a decoupled-level with QoS-aware downlink scheduling algorithm for LTE networks. In Proceedings of the 2015 IEEE International Conference on Data Science and Data Intensive Systems, Sydney, Australia 11-13 December 2015; pp. 696-704.

66. Piro, G.; Grieco, L.A.; Boggia, G.; Fortuna, R.; Camarda, P. Two-level downlink scheduling for real-time multimedia services in lte networks. IEEE Trans. Multimed. 2011, 13, 1052-1065. [CrossRef]

67. Sandrasegaran, K.; Mohd Ramli, H.A.; Basukala, R. Delay-Prioritized Scheduling (DPS) for real time traffic in 3GPP LTE system. In Proceedings of the 2010 IEEE Wireless Communication and Networking Conference, Sydney, Australia, 18-21 April 2010; pp. 1-6.

68. Skondras, E.; Michalas, A.; Sgora, A.; Vergados, D.D. A downlink scheduler supporting real time services in LTE cellular networks. In Proceedings of the 2015 IEEE International Conference on Information, Intelligence, Systems and Applications, Corfu, Greece, 6-8 July 2015; pp. 1-6.

69. Samie, H.; Moulay, E.; Coirault, P.; Vauzelle, R.; Launay, F. Potential feedback control for data scheduling in LTE cellular networks. In Proceedings of the 2017 IEEE International Conference on Information, Intelligence, Systems \& Applications, Larnaca, Cyprus, 27-30 August 2017; pp. 1-6.

70. Samia, D.; Ridha, B.; Wei, A. Resource allocation using nucleolus value in downlink LTE networks. In Proceedings of the 2016 IEEE Symposium on Computers and Communication, Messina, Italy, 27-30 June 2016; pp. 250-254.

71. Ali, S.; Zeeshan, M. A delay-scheduler coupled game theoretic resource allocation scheme for LTE networks. In Proceedings of the 2011 IEEE Frontiers of Information Technology, Islamabad, Pakistan, 19-21 December 2011; pp. 14-19.

72. Ferdosian, N.; Othman, M.; Lun, K.Y.; Ali, B.M. Optimal solution to the fractional knapsack problem for LTE overload-state scheduling. In Proceedings of the 2016 IEEE International Symposium on Telecommunication Technologies, Kuala Lumpur, Malaysia, 28-30 November 2016; pp. 97-102.

73. Ferdosian, N.; Othman, M.; Ali, B.M.; Lun, K.Y. Multi-targeted downlink scheduling for overload-states in LTE networks: Proportional fractional knapsack algorithm with Gaussian weights. IEEE Access 2017, 5, 3016-3027. [CrossRef]

74. Astudillo, C.A.; de Andrade, T.P.C.; da Fonseca, N.L.S. Allocation of control resources with preamble priority awareness for human and machine type communications in LTE-Advanced networks. In Proceedings of the 2017 IEEE International Conference on Communications, Paris, France, 21-25 May 2017; pp. 1-6.

75. Wang, P.; Song, W.; Niyato, D.; Xiao, Y. QoS-aware cell association in 5G heterogeneous networks with massive MIMO. IEEE Netw. 2015, 29, 76-82. [CrossRef]

76. Bliss, D.; Forsythe, K.; Fawcett, G. MIMO Radar: Resolution, performance, and waveforms. In Proceedings of the 14th Annual Adaptive Sensor Array Processing Workshop, Lexington, MA, USA, 6-7 June 2006; pp. 6-7.

77. Hwang, T.; Yang, C.; Wu, G.; Li, S.; Li, G.Y. OFDM and its wireless applications: A survey. IEEE Trans. Veh. Technol. 2009, 58, 1673-1694. [CrossRef]

78. Al-Jzari, A.; Iviva, K. Cyclic prefix length determination for orthogonal frequency division multiplexing system over different wireless channel models based on the maximum excess delay spread. Am. J. Eng. Appl. Sci. Orig. Res. Paper 2015, 8, 82-93. [CrossRef]

79. Nascimento, A.; Gameiro, A. Jointly cross-layer scheduling and dynamic resource allocation for RT and NRT traffic types for IEEE802.16e. In Proceedings of the 2009 IEEE Vehicular Technology Conference, Barcelona, Spain, 26-29 April 2009; pp. 1-6. 
80. Calabuig, D.; Monserrat, J.F.; Gomez-Barquero, D.; Cardona, N. A Delay-Centric Dynamic Resource Allocation Algorithm for Wireless Communication Systems Based on HNN. IEEE Trans. Veh. Technol. 2008, 57, 3653-3665. [CrossRef]

81. Cormen, T.H.; Leiserson, C.E.; Rivest, R.L.; Stein, C. Introduction to Algorithms, 3rd ed.; The MIT Press: Cambridge, MA, USA, 2009.

82. Zarrinkoub, H. Understanding LTE with MATLAB: From Mathematical Modeling to Simulation and Prototyping; Wiley: Hoboken, NJ, USA, 2014.

83. 3GPP 36 Series TS 136 101V10.x-Evolved Universal Terrestrial Radio Access; User Equipment Radio Transmission and Reception. Available online: https://www.etsi.org/deliver/etsi_ts/136100_136199/ 136101/10.03.00_60/ts_136101v100300p.pdf (accessed on 14 November 2018).

84. Jain, R.; Chiu, D.M.; Hawe, W.R. A Quantitative Measure of Fairness and Discrimination for Resource Allocation in Shared Computer System; DEC Technical Report TR301; Digital Equipment Corporation: Hudson, MA, USA, 1984; pp. 1-38.

85. Estella, I.; Pascual-Iserte, A.; Payaro, M. OFDM and FBMC performance comparison for multistream MIMO systems. In Proceedings of the 2010 Future Network \& Mobile Summit, Florence, Italy, 16-18 June 2010; pp. 1-8.

86. Zhang, H.; Le Ruyet, D.; Roviras, D.; Medjahdi, Y.; Sun, H. In Proceedings of the Spectral efficiency comparison of OFDM/FBMC for uplink cognitive radio networks. Eurasip J. Adv. Signal Process. 2010, 2010, 1-15. [CrossRef]

87. He, Q.; Schmeink, A. Comparison and evaluation between FBMC and OFDM systems. In Proceedings of the WSA 2015; 19th International ITG Workshop on Smart Antennas, Ilmenau, Germany, 3-5 March 2015.

88. Zabini, F.; Bazzi, A.; Masini, B.M.; Verdone, R. Optimal performance versus fairness tradeoff for resource allocation in wireless systems. IEEE Trans. Wirel. Commun. 2017, 16, 2587-2600. [CrossRef]

89. Lan, T.; Kao, D.; Chiang, M.; Sabharwal, A. An axiomatic theory of fairness in network resource allocation. In Proceedings of the 2010 IEEE INFOCOM, San Diego, CA, USA, 14-19 March 2010.

(C) 2018 by the authors. Licensee MDPI, Basel, Switzerland. This article is an open access article distributed under the terms and conditions of the Creative Commons Attribution (CC BY) license (http:/ / creativecommons.org/licenses/by/4.0/). 